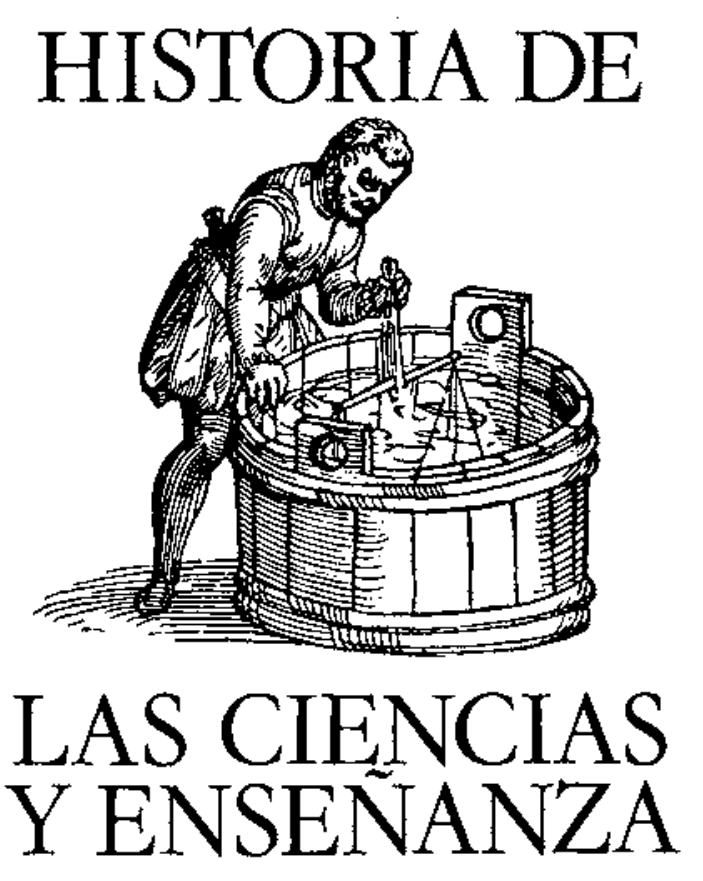

\title{
CÓMO UTILIZAR LA HISTORIA DE LAS CIENCIAS EN LA ENSEÑANZA DE LAS CIENCIAS
}

GAGLIARDI, R.

L.D.E.S. Universidad de Ginebra.

\section{SUMMARY}

Some elements justifying the use of Science History and Epistemology, with no increase of the amount of information for the students, are offered.

\section{INTRODUCCIÓN}

Vamos a dar algunos elementos para justificar la utilización de la historia de las ciencias y la epistemologia en la enser̃anza de las ciencias. Nuestra intención no es aumentar la cantidad de información que reciben los alumnos, ni someterios a clases más largas y difíciles, sino comenzar una discusión que creemos fructifera sobre los modos posibles de mejorar la enseñanza de las ciencias en la escuela. 
Muchas experiencias pedagógicas demuestran que en la mayor parte de los casos analizados la enseñanza de las ciencias no da buenos resultados, los alumnos no aprenden, o aprenden parcialmente los conocimientos científicos que la escuela trata de transmitirles.

Este fracaso no es un fenómeno aislado: a diferentes niveles escolares, en diferentes países, en medios sociales diferentes, el análisis de lo que los alumnos conocen después de finalizados los estudios muestra que lo que se recuerda es poco y es equivocado. Pero aún, en muy pocos casos se construyen las estructuras cognitivas que permiten continuar el aprendizaje de las ciencias.

En otros términos, la población no se apropia de los conocimientos científicos que podrían ser útiles para mejorar la calidad de vida, ni es capaz de comprender exactamente cuáles son los problemas que plantea la producción, el control y la utilización de esos conocimientos.

Todo eso ha sido muy debatido en diversos congresos, y las soluciones propuestas por la pedagogia de ciencias -en particular la utilizactón del análisís de representaciones de los alumnos- están siendo aplicadas por muchos enseriantes con un resultado positivo.

En ese marco de análisis crítico de la enseñanza de ciencias se inscribe la proposición de utilizar la historia de las ciencias y la epistemología, como un complemento de las áreas más desarrolladas de la pedagogia de ciencias.

La historia de las ciencias y de la epistemología pueden ser utilizadas en la enseñanza de ciencias de diversas maneras:

- Para la determinación de obstáculos epistemológicos.

- Para la definición de contenidos de la enseñanza.

- Para introducir en clase la discusión sobre la producción, la apropiación y el control de los conocimientos a nivel social e individual.

- Como complemento de la enserfanza de otras disciplinas, en particular la historia y la geografia.

Estas posibilidades no son exclúyentes de otras que se pueden ir desarrollando en la medida que la historia de las ciencias y la epistemología se introduzcan en la escuela.

\section{LA HISTORIA DE LAS CIENCIAS Y LA EPISTEMOLOGÍA COMO UNA HERRA- MIENTA PARA LA DETERMINACIÓN DE OBSTACULOS EPISTEMOLÓGICOS}

Actualmente uno de los objetivos más importantes de la pedagogía de las ciencias es lograr que el enseñante ayude concretamente al alumno a superar los obstácu- los a la construcción de conocimientos.

Esto significa terminar con la repetición de informaciones que no pueden ser comprendidas por el alumno, y comenzar a establecer las estrategias y los contenidos que permita al alumno realizar un trabajo cognitivo y poder superar los obstáculos del aprendizaje.

Sin entrar en un debate sobre la psicologia, y sabiendo que toda simplificación es un error parcial, podemos decir que existen tres tipos de obstáculos en el aprendizaje de ciencias:

- Los obstáculos derivados del desarrollo de la inteligencia, que podemos llamar obstáculos lógicos, y que han sido tratados por la psicología genética.

- Los obstáculos derivados de problemas afectivos o psicológicos, como el rechazo a la clase, la desvalorización del alumno, los tabús, etc., que han sido tratados por el psicoanálisis.

- Los obstáculos derivados de la estructura del sistema cognitivo, que Ilamaremos obstáculos epistemológicos.

Es importante recalcar que esta clasificación se realiza para organizar nuestro discurso, pero que en realidad la incapacidad de construjr un nuevo conocimiento es necesariamente lógica, afectiva y epistemológica, pues no se pueden separar esos tres niveles. Sin embargo creemos que el tratar específicamente los obstáculos epistemológicos puede ser una ayuda para resolver concretamente los otros dos tipos de obstáculos: el aiumno que logró transformar su sistema cognitivo transforma su estructura lógica (no existen estructuras sin contenidos) y suele mejorar la imagen de sí mismo, lo cual es importante para superar muchos obstáculos afectivos.

La determinación de los obstáculos epistemológicos es uno de los aspectos más importantes de la transformación de la enseñanza de ciencias (y de toda la enseñanza), pues permite encarar la enseñanza desde el ángulo de la construcción de conocimientos y no de la memorización de informaciones, lo que significa centrar la actividad en el desarrollo de la capacidad de aprender. En esa perspectiva, el alumno debe transformar su sistema cognitivo en función de lo que aprende. Los resultados del aprendizaje se miden en función de la transformación de los propios alumnos y no en función de la masa de conocimientos que pueden memorizar.

Lo que importa es que los alumnos hayan superado los obstáculos que impedian la construcción de nuevos conocimientos, lo que les permite continuar su aprendizaje después de los estudios. La escuela ya no es «el lugar donde se aprende ciencia», sino «el lugar donde se transforma el sistema cognitivo para poder aprender ciencia).

Determinar cuáles con los principales obstáculos epistemológios significa poder medir la transformación 
conceptual de los alumnos, y poder establecer curriculos flexibles, que pueden modificarse en función del tiempo empleado para superar obstáculos más importantes.

El análisis de representaciones es un instrumento eficaz para determinar los obstáculos epistemológicos de los alumnos, pero requiere conocer bien la ciencia que se enseña, y, además, conocer su historia.

La historia de las ciencias permite comprender cuáles son las principales teorías actuales y cuáles han sido los obstáculos que trabaron su aparición y el desarrollo de una ciencia.

Esto no significa postular un "paralelismo» entre la historia de las ciencias y el desarrollo de la inteligencia y del conocimiento individual. La historia de las ciencias puede dar «pistas» pero no elimina el análisis concreto de los alumnos concretos en una situación concreta, para hablar concretamente. Queremos decir con esto que el alumno actual vive, piensa, construye sus conocimientos en una sociedad diferente en la cual se produjeron los conocimientos que debe reproducir en clase. Es decir que no es similar a los cientificos. Sin embargo, está claro que conocer cuáles fueron las trabas para desarrollar socialmente ciertos conocimientos puede ser muy útil para comprender las dificultades de los alumnos.

Tomando un ejemplo de la biología, el desconocimiento de la célula impide comprender aspectos fundamentales del funcionamiento de los organismos vivos. $\mathrm{Pa}-$ ra construir la noción de células no bastaron las observaciones microcópicas: transcurrieron más de doscientos años entre las primeras descripciones de células y la elaboración de la teoría celular. ¿Por qué esa demora? ¿Por qué la «moda de la observación microscópica» no se tradujo en una teoría celular?

Para poder construir dicha teoría era necesario comprender que las propiedades macroscópicas de los organismos dependían de su estructura microscópica. En términos de obstáculos epistemológicos, era necesario superar el «obstáculo macroscópico» para poder integrar las observaciones en una totalidad coherente que permitiera elaborar una teoria explicativa global.

El andulisis de representaciones (Gagliardi et al. 1987), seriala que los alumnos no construyen un concepto operativo de célula, es decir que tienen idea de la existencia de las células pero no piensan que su organismo esté en realidad formado de células, lo siguen viendo sólo como una entidad macroscópica. Una vez que la historia de las ciencias y el análisis de representaciones se conjugan, es posible desarrollar una clase centrada sobre el obstáculo macroscópico y lograr que los alumnos desarrollen el concepto de célula, no como una simple información, sino como un elemento para construir ntevos conocimientos.

En una clase en Barbate (Martínez Oros y Gagliardi 1987), se desarrolló un modelo de enseñanza de la fi- siología y la anatomía humanas fundado en la noción de «centros de crecimiento distribuidos por todo el organismo", como una noción esencial para desarrollar la idea de célula y la necesidad del transporte de sustancias del sistema digestivo a todo el organismo. Obtuvo excelentes resultados: alumnos de nueve años comprendieron las nociones propuestas y continuaron desarroliando su pensamiento hasta proponer espontáneamente la existencia del sistema circulatorio y la existencia de una "bomba mecánica» (el corazón) que impulsaba la sangre. Esos alumnos conservaron en los años sucesivos esos conocimientos.

En una escuela de Italia, en Pavía, después de estudiar las representaciones sobre la célula (Gagliardi et al. 1987) se organizó un curso sobre anatomía fundado en la toma de consciencia del movimiento y la nutrición necesaria. Se obtuvo que sus alumnos de nueve y diez años llegaran a plantearse el pasaje de los alimentos por la barrera intestinal.

En otros términos, una vez que los alumnos comprenden que su crecimiento es resultado de la incorporación de nuevas sustancias que deben ser transportadas a todo el organismo, desarrollan lógicamente un esquema de transporte (sistema circulatorio), de transformación en el sistema digestivo y de pasage de ciertas barreras.

Queremos mostrar con esto que la utilización de una metodología de análisis de representaciones, sumada al estudio de los principales momentos de la historia de una ciencia puede permitir organizar la clase de modo que los alumnos desarrollen nuevas estructuras cognitivas que les permiten construir nuevos conocimientos $\sin$ grandes dificultades, $\mathrm{y}$, lo que es más importante, que desarrollen nuevos esquemas que les permiten construir nuevos conocimientos como resultado de deducciones lógicas.

\section{UTILIZACIÓN DE LA HISTORIA DE LAS CIENCIAS Y DE LA EPISTEMOLOGIA PARA DEFINIR LOS CONTENIDOS DE LOS CURSOS}

Este punto no es más que un desarrollo del punto anterior. En efecto, nuestra proposición es centrar los cursos en los conceptos estructurantes, es decir en aquellos conceptos que una vez que han sido construidos por el alumno determinan una transformación de su sistema conceptual que permite continuar con el aprendizaje (Gagliardi 1986). Una enseñanza fundada en los conceptos estructurantes reduce los temas a enseriar y permite dedicar más tiempo al desarrollo de la capacidad de los alumnos. En otros términos, los conceptos estructurantes son a la vez un medio para superar los obstáculos epistemológicos y una base para continuar aprendiendo. 
Los conceptos estructurantes deben ser determinados a partir del análisis de las teorías científicas actuales y de su historia. La historia de las ciencias permite visualizar cuáles han sido los conceptos que han permitido la transformacion de una ciencia, la elaboración de nuevas teorias, la utilización de nuevos métodos y nuevos instrumentos conceptuales.

Al mismo tiempo esto presupone una concepción epistemológica que ve el desarrollo de la ciencia como resultado de transformaciones estructurales profundas (Kuhn 1983), que no sólo cambian las teorías, sino que «determinan nuevos observables». La "realidad a analizar» cambia en función de los cambios conceptuales, nuevos fenómenos son tomados en consideración. Siguiendo con el ejemplo anterior, elaborar el concepto de relación entre los fenómenos macroscópicos y el sustrato microscópico determinó la aparición de nuevos problemas a resolver en la física (estructura de la materia), la química (composición de las sustancias) y la biología (estructura celular). Esos problemas no existían antes de que fuera elaborado ese concepto.

Como hemos visto en los ejemplos de clases mencionados en el punto 1, los alumnos «descubren nuevos problemas a resolver " cuando construyen ciertos conceptos, y esos problemas los motivan a seguir aprendiendo.

\section{UTILIZACIÓN DE LA HISTORIA DE LAS CIENCIAS Y DE LA EPISTEMOLOGÍA PARA INTRODUCIR EN CLASE LA DISCUSIÓN SO- BRE LA PRODUCCIÓN. LA APROPIACIÓN Y EL CONTROL DE LOS CONOCIMIENTOS.}

En los puntos anteriores hemos tratado la posibilidad de utilizar la historia de las ciencias y de la epistemologia para definir programas de estudio y estrategias pedagógicas. Vamos a tratar ahora la necesidad de introducir esas disciplinas como temas especificos de los cursos de ciencias.

Enseñar la bistoria de las ciencias y la epistemología en la escuela no parece una tarea fácil. A las dificultades ya existentes de los cursos de ciencias se suma la introducción de nuevos temas, la fatiga de los estudiantes a comprender los procesos históricos, el escaso tiempo disponible que habitualmente no alcanza para completar los largos programas de ciencias. Todo esto es cierto desde una perspectiva "tradicional», en la cual se trata de lograr que los alumnos aprendan el máximo posible. Pero las cosas cambian desde una nueva perspectiva de la enseffanza de las ciencias (y de la ensefianza en general), que se está tratando de elaḅorar en colaboración con muchos ensenantes de ciencias de diversos países. Esa perspectiva postula una enseñanza que parte de las representaciones de los alumnos y trata de lograr que éstos superen los obstáculos al aprendizaje mediante la construcción de ciertos con- ceptos estructurantes. En esa perspectiva no hay largos programas a desarrollar, sino un esquema flexible, que permite al enseñante adecuarse al proceso de aprendizaje concreto de sus alumnos, dándoles el tiempo necesario para desarrollar sus capacidades

Si la idea es reducir la ensenianza a lo esencial, por qué planteamos introducir nuevos temas históricos y epistemológicos?

Por dos motivos esenciales:

- La utilización de la historia de las ciencias y de la epistemología para comprender la sociedad humana.

- La utilización de la historia de las ciencias y de la epistemología para comprender los mecanismos de producción y la reproducción social e individual de conocimientos.

El primer motivo está fundamentado en la relación entre el desarrollo de nuevas técnicas de transformación de la naturaleza. Las transformaciones que sufrió y sufre la sociedad humana están estrechamente relacionadas con los procesos de apropiación de los recursos disponibles. Eso no quiere decir que la ciencia ha transformado la sociedad, ni que el desarrollo de la ciencia esta sólo determinado por la estructura social. Creemos que es necesario superar los diversos reduccionismos y tratar de entender el funcionamiento de un sistema tan complejo, con tantas interacciones, como es la sociedad humana.

Es muy importante a nuestro juicio lograr que los alumnos comprendan esa complejidad y puedan manejarla, lo que significa una visión que integra la historia, la economía, la geografía, la tecrología y la ciencia como parte de un sistema complejo de producción y reproducción de conocimientos y técnicas de transformación de la naturaleza

El segundo motivo de la introducción de la historia de ciencias y de la epistemología en los cursos de ciencias es promover una discusión sobre los mecanismos de construcción y reproducción de conocimientos, a nivel del propio alumno y a nivel de la sociedad.

Resulta paradojico que en la escuela se traten muchisimos temas, pero el conocimiento en si mismo no sea un tema de clase. Creemos que el alumno debe ser consciente de los mecanismos que utiliza para elaborar su pensamiento, y de los procesos sociales que llevan a la elaboración de nuevas teorias científicas. En particular nos parece muy importante que los alumnos tomen consciencia de que reproducen conocimientos ya elaborados socialmente, pero que los producen por un proceso individual de re-construcción. En ese sentido la historia de las ciencias y la epistemología permiten ver las diferencias entre los procesos individuales y los procesos institucionales de construcción de conocimientos.

Un punto que merece una discusión es la diferencia entre la observacion empírica que pueden realizar los 
alumnos y la elaboración de teorías cientificas. Los alumnos no hacen ciencia en clase. La ciencia es una actividad institucional, integrada, en la que se aceptan implicitamente o explícitamente ciertas teorias previas, y en la cual se utilizan métodos aceptados por la comunidad científica. La actividad en clase de los alumnos no es similar a la actividad cientifica. La discusión de la epistemología puede ser un medio para superar esa confusión relativamente frecuente en los enseñantes de ciencias.

\section{LA HISTORIA DE LAS CIENCIAS COMO COMPLEMENTO DE OTRAS DISCIPLINAS (EN PARTICULAR LA HISTORIA Y"LA GEOGRAFÍA)}

La enseñanza de la historia de las ciencias aparece como un complemento necesario a la enseñanza de la historia, en la medida en que, como hemos señalado, la construcción de conocimientos y su aplicación a la tecnologia son parte importante de las transformaciones sociales. En ese sentido también es útil ligar la historia de las ciencias a la geografía para mostrar las transformaciones del territorio provocadas por la utilización de esas técnicas y los límites que el propio territorio impone

Otro argumento para relacionar la historia de las ciencias con las ciencias sociales es la importancia cada vez mayor de la ciencia en la sociedad actual, y las transformaciones que se producen a nivel de la apropiación de los conocimientos cientificos

La ciencia es una actividad financiada por el conjunto de la población, que es utilizada para generar riquezas y poder en beneficio de un pequeño sector de la sociedad. Una sociedad democrática requiere que la población disponga de los medios necesarios para controlar la producción y la utilización de conocimientos científicos. Esto significa que la población debe comprender lo que está en juego, saber cuáles son las alternativas, tener la capacidad de decidir con conocimiento de causa. Todo esto puede lograrse si la escuela desarrolla la capacidad de comprensión de los mecanismos sociales que llevan a la apropiación de los conocimientos científicos y los riesgos que implica su utilización.
Ver, por ejemplo, como se pasa de la producción y apropiación social de conocimientos a la producción institucional y apropiación individual es un paso importante para comprender la transformación de la sociedad

La epistemología puede permitir, además, mostrar cómo se reproducen y se unaturalizan" las ideas dominantes en la sociedad, es decir como se construye y se reproduce la ideología dominante. Finalmente la epistemología puede permitir clarificar los mecanismos de justificación de un modelo de sociedad por el desarroIlo de la ciencia y la tecnologia (Habernas 1978), que son uno de los modos de justificar la sociedad actual. En un momento en que se discute la continuación de ciertas técnicas que ponen en peligro la vida sobre el planeta, la epistemología puede ser un medio de capacitar a los alumnos a intervenir conscientemente en ese debate.

Finalmente la introducción de la historia de las ciencias y la epistemología puede permitir un debate sobre la propia estructura de la ciencia actual, sus relaciones con el poder, sus modos de operar, tas teorías dominantes, etc. Ese debate fundamental para comprender el funcionamiento de una de las instituciones más importantes del mundo moderno, puede permitir «desmitificar» la ciencia y aumentar las posibilidades del alumno de participar en la construcción y en el control de los conocimientos.

\section{SÍNTESIS}

Hemos tratado de dar algunos elementos que justifican la utilización de la ciencia y de la epistemología en la enseñanza de las ciencias, en la perspectiva de una transformación de los sistemas de enseñanza que permita una participación consciente de Ios alumnos en la construccion de sus conocimientos. No creemos que este debate se cierre con el enunciado de esta hipótesis. Al contrario, creemos que será necesario un largo camino para «inventar» la nueva pedagogía. La historia de las ciencias puede servirnos para comprender la importancia de la paciencia y la persistencia para lograr que una idea sea aceptada por las instituciones. 


\section{REFERENCIAS BIBLIOGRÁFICAS}

GAGLIARDI, R., 1986, Los conceptos estructurales en el aprendizaje por investigacion, Enseñanza de las Ciencias, 4, Vol. 1, pp. 30-35.

GAGLIARDI, R., MOSCONI BERNARDINI, P., BOCCIOLA, M.T., STRUSBERG, S., SIMEONE, R. y SIMEONE, L., 1987, Nous ne sommes pas de petits ballons. Les modèles spontanés des élèves de l'école italienne sur la cellule, Actes des IXèmes Journées Internationales sur l'Educacion Scientifique.
HABER NAS, J., 1978, La Technique et la science comme idéologie. La fin de la métaphysique. (Denoël/Gonthier: Paris).

KUHN, T.S., 1983, La structure des revolutions scientifiques. (Flammarion: Paris).

MARTÍNEZ OROZ, C. y GAGLIARDI, R., 1987, Les modèles sur la croissance et leur utilisation dans l'enseignement. Actes des IXèmes Journées Internationales sur I'Education Scientifique. 